\title{
Interface conditions for fast-reaction fronts in wet porous mineral materials: the case of concrete carbonation
}

\author{
A. Muntean · M. Böhm
}

Received: 20 September 2007 / Accepted: 30 April 2009 / Published online: 17 May 2009

(C) The Author(s) 2009. This article is published with open access at Springerlink.com

\begin{abstract}
Reaction-diffusion processes, where slow diffusion balances fast reaction, usually exhibit internal loci where the reactions are concentrated. Some modeling and simulation aspects of using kinetic free-boundary conditions to drive fast carbonation reaction fronts into unsaturated porous cement-based materials are discussed. Providing full control on the velocity of the reaction front, such conditions offer a rich description of the coupling between transport, reaction, and change in the shape of the a priori unknown time-dependent regions. New models are formulated and validated by means of numerical simulations and experimental data.
\end{abstract}

Keywords Concrete carbonation $\cdot$ Fast-reaction fronts $\cdot$ Modeling $\cdot$ Reaction-diffusion systems $\cdot$ Stefan problem

\section{Introduction}

A variety of reaction-diffusion problems, which are common in physics, chemistry or biology involve the propagation of moving internal sharp reaction interfaces or thin strips (see, for instance, [1-3]). This happens whenever there is an intrinsic mechanism that enforces a spatial segregation of the dominant characteristic length scales. In the reaction-diffusion scenarios we consider, the separation boundary and its internal structure result from the balancing of diffusion and reaction. The diffusive effect, which smoothes and mixes the properties of the material, competes with the mechanism of reaction that drives the reaction locus out of thermodynamic equilibrium. When spatially separated reactants meet, a reaction zone forms at the separation boundary. If the reaction is very fast, the reaction zone is usually narrow. In such a setting, the characteristic reaction time scale is much smaller than that of diffusion.

A wealth of similar problems can be found in geochemistry [1,4], phase transitions in steels [5], material processing or corrosion problems in civil engineering [6-9]. The common feature of these scenarios is that the reaction zone is driven by means of kinetic laws-explicit descriptions of the normal component of the velocity

A. Muntean $(\varangle)$

CASA - Centre for Analysis, Scientific Computing and Applications, Department of Mathematics and Computer Science,

Technical University of Eindhoven, Eindhoven, The Netherlands

e-mail: a.muntean@tue.nl

\section{Böhm}

Centre for Industrial Mathematics (ZeTeM), Department of Mathematics and Computer Science, University of Bremen, Bremen, Germany

e-mail: mbohm@math.uni-bremen.de 
of the moving front as a function of reactant concentrations. Consequently, we are always led to coupled quasilinear partly dissipative systems of non-homogeneous parabolic equations permitting the presence of fixed and/or moving internal boundaries. The use of kinetic conditions has both advantages and drawbacks. On the one hand, the kinetics often prevents the blow-up of the solution in finite time, while on the other the fronts are expected to move continuously (without any plateaux in the velocity-vs.-time profile) possibly leading to an over-estimation of the final time of the process [8-10]. In this paper, we point out a couple of nonlinear behaviors for one of the most important durability issues in civil engineering - the concrete-carbonation problem-when kinetic conditions drive surface-concentrated reactions. More details on the approach can be found in [9].

The plan of the paper is as follows. In Sect.2, we concisely describe the underlying physical and chemical processes as well as the geometry which our problem refers to. In Sects. 3 and 4, we discuss a couple of nonequivalent possibilities for defining the position of the moving front and derive a kinetic law for triggering it into the concrete. Note that everywhere in this paper the reaction front is assumed to be sufficiently thin, that is, either we deal with a sharp interface or with a thin strip. Section 4.2 contains the complete list of model equations describing this scenario. Simulation examples illustrate the behavior of the sharp-interface model in Sect. 5. We also point out a few open modeling issues regarding $2 \mathrm{D}$ moving-boundary approaches to the carbonation problem.

\section{Kinetic moving-boundary problems applied to concrete carbonation}

Carbon dioxide from the surrounding environment penetrates concrete structures via the air-filled parts of the pores, dissolves in the pore water and reacts there with the available dissolved calcium hydroxide coming from the solid matrix. The basic assumption for this process to happen is that the porous material is sufficiently wet but not saturated. For a reduced scenario, the carbonation reaction can be written in the form

$\mathrm{CO}_{2}+\mathrm{Ca}(\mathrm{OH})_{2} \rightarrow \mathrm{H}_{2} \mathrm{O}+\mathrm{CaCO}_{3}$.

The chemistry of this process is much more complex than stated in (1); see [12,14,15] for more details on this civil engineering issue. Although only the case of a single chemical reaction will be discussed here, the methodology can be applied to situations involving more complex chemistry. The reaction-diffusion process that is associated with (1), also called concrete carbonation, takes place in all concrete-based materials exposed to air. It has a major impact on these materials especially because it destroys their alkalinity (which protects the steel reinforcement against corrosion) [12,18] and alters the micro-structure [17]. In carbonated concretes like bridges, parking houses, buildings in a marine environment, corrosion initiates easily due to the action of aggressive chemicals. Hence, spalling and other unwanted effects due to corrosion can occur. Although the phenomenology of the overall process is well known, there are several qualitative and quantitative behaviors connected to concrete carbonation (especially under natural exposure conditions) which are not fully understood, and hence, the accuracy of current quantitative predictions of penetration depths is questionable; see e.g. [9,21]. In what we are concerned with two basic questions need to be answered:

(a) Why and how is a moving-boundary (interface) approach applicable to carbonation scenarios?

(b) Which mechanism drives the reaction location?

The reader can find details on question (a) in $[9,11,17,22]$. Within this framework, our attention is rather focused on answering question (b). The expected result is twofold: (1) to get at a better understanding of the process; and

(2) to gain better quantitative predictions of penetration depths. We show in Sect. 4 that the right answer to question (b) provides at the same time a good estimate of the time needed to initiate corrosion.

Recently, the carbonation process received renewed attention from the modeling perspective. Conceptually different models have been developed in order to study the carbonation penetration in unsaturated concrete samples, viz:

(1) moving sharp-interface models;

(2) moving reaction-strip models; 
(3) moving two-reaction-zones models;

(4) isolines models.

Our main contribution consists in developing non-equilibrium models of types 1-3 (see [8,22] and [9]) and their testing against models of type $4([17,23])$. We refer to the models of types $1-3$ as MBP (Moving-Boundary Problems). In the sequel, we present the basic methodology on which the non-equilibrium models of the types 1-3 rely; we derive a kinetic free-boundary condition and address the questions (a) and (b) from the perspective of MBP models.

For models of types 1-3, the model output consists of both concentration profiles and position of the localized reaction, while for that of type 4 the output contains only concentration profiles. In models of type 4, the position of the localized reaction is defined a posteriori using the concept of degree of reaction or other similar notions [25].

\section{What is the carbonation front?}

The first question that needs to be answered is a seemingly trivial one: What is the carbonation front? or, in other words, What is the right definition of the position of the carbonation front? At this moment, there is a lack of consensus in the civil engineering literature in answering such a question. It is worth noting that there are a few conceptually different alternative definitions, which may be taken into account. For instance, one way to define the position of the front is to employ the chemistry of the sample. In this sense, one can estimate the drop in $\mathrm{pH}$, which can be nicely visualized by spraying phenolphthalein on cross-sections of partially carbonated samples [21], or one can take this information by estimating the steepness of a reactant profile [17] or calculating a reaction degree $[18,25]$. Carbonation models of type 4 rely on this kind of definitions. A distinct way to tackle this issue is to define the position of the reaction front using the geometry of the reaction front itself and consider it as an additional unknown in the model. This is a typical situation which may be dealt with via moving-boundary approaches describing fast-reaction-slow-diffusion scenarios. Furthermore, it is often a matter of assumption whether a reaction front is a sharp interface or a thin strip. ${ }^{1}$ We distinguish between these two conceptually different cases as follows (see Fig. 1 for a sketch of a one-dimensional geometry we are investigating):

(A) the reaction front associated with (1) is thin and can be idealized by a sharp interface $\Gamma(t)$ positioned at $x=s(t)$;

(B) the reaction is localized within a strip $\Omega_{\epsilon}(t)$ of width $\epsilon$, whose center line $\Gamma(t)$ is positioned at $x=s(t)$. $\Omega_{\epsilon}(t)$ cannot be approximated by its center line $\Gamma(t)$.

The carbonation problem presented as in case (A) was initially discussed two decades ago by Tuutti [26], and afterwards, by Brieger and Whitmann (they simply employed the Neumann solution of a two-phase Stefan problem; see [27]). A generalization to more complex reaction-diffusion scenarios has been recently developed in [22]; for a literature review, see [9, Sect. 2.1.2].

The approach indicated in case (B) is conceptually new. Although the analysis and simulation of this model (of type 2 or 3 ) are nowadays relatively standard, the modeling part is more involved and far from being complete. The difficult part is that two essential pieces of information are always missing:

(a) the a priori width of the strip;

(b) how fast a reaction strip advances into the material. It is worth emphasizing that, although one knows quite a $\operatorname{lot}^{2}$ about the velocity of sharp reaction interfaces, far less is known if reaction strips are about to move.

A way to control the unwanted effects produced by small variations in (a) and (b) is to provide stability estimates (for both the free boundary and the concentration profiles) with respect to parameters, initial and boundary data. For

\footnotetext{
${ }^{1}$ Generally, in applications the reaction front is not sharp.

2 At least for simple reaction-diffusion scenarios like those describing the melting of ice bowls (the classical Stefan problem cf. [27-29], e.g.), solidification of binary alloys [5,30], or flame propagation in combustion problems $[19,20]$, one can estimate the right size of the speed of the moving sharp interface.
} 


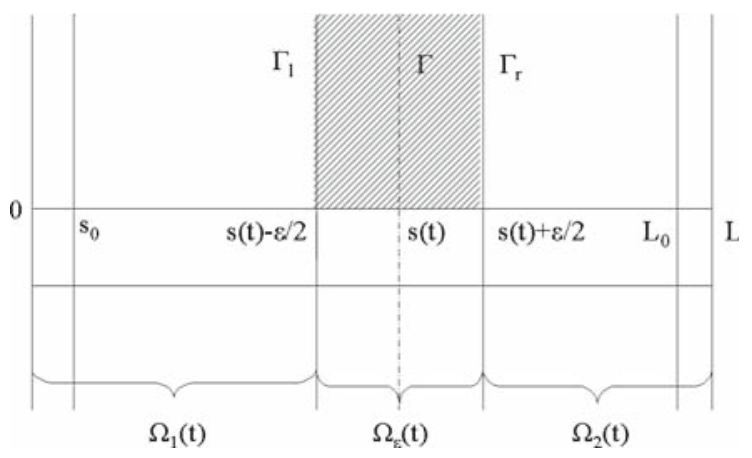

Fig. 1 Basic geometry in $1 \mathrm{D}$ case. $\Omega_{1}(t):=(0, s(t)-\epsilon / 2)$ is the carbonated zone, $\Omega_{\epsilon}(t):=(s(t)-\epsilon / 2, s(t)+\epsilon / 2)$ is the strip where reaction (1) is concentrated, and $\Omega_{2}(t):=(s(t)+\epsilon / 2, L)$ is the uncarbonated part. If the reaction concentrates on a surface (i.e., $\epsilon=0)$, then $\Omega_{\epsilon}(t)$ degenerates to the interface $\Gamma(t) . L_{0}$ is an arbitrary depth in $(0, L)$

the carbonation problem, such stability estimates have been derived in [9] (Theorems 3.4.8 and 3.5.5). In particular, they state that small changes in the parameters defining the reaction rate of (1) induce small changes in the model output.

\section{How fast can a reaction strip move?}

We can only give an incomplete answer to the question of how fast a reaction strip can penetrate a given material (here: part of the concrete sample). The general answer to this question appears to be an open problem at the modeling level. In this section, we focus on a simple situation and derive a law for the normal component of the velocity of a sharp interface. Next, relying on the structure of the latter law, we suggest an ansatz of the normal component of the velocity of a straight thin strip.

\subsection{Derivation of the kinetic condition}

Let $\Omega$ be a slab and $\Gamma(t)$ the moving plane where reaction (1) is concentrated. We consider the advancement of the flat surface $\Gamma(t)$ over a time interval $S:=(t, t+\Delta t] \subset S_{T}=[0, T]$ into an arbitrary region $\Omega^{\prime} \subset \Omega$. Additionally, we assume that behind the plane $\Gamma(t)$ the reaction (1) is complete. The mass concentration of $\mathrm{Ca}(\mathrm{OH})_{2}$ at the pore level is denoted here by $H$. Furthermore, let $\phi$ be the concrete porosity and $\phi_{w}$ the water fraction. If $\Omega^{\prime}$ is the region between the planes $\Gamma(t)$ and $\Gamma(t)+\Delta s$, where $\Delta s$ is a small increment in space, then the amount of $\mathrm{Ca}(\mathrm{OH})_{2}$ consumed during $S$ in $\Omega^{\prime}$ is given by $\Delta s \phi \phi_{w} H\left(x, t^{\prime}\right)\left|\Gamma\left(t^{\prime}\right)\right|$ for some $t^{\prime} \in(t, t+\Delta t]$ (before carbonation). Here $\left|\Gamma\left(t^{\prime}\right)\right|$ is the area of the surface $\Gamma$ at time $t^{\prime}$. After a time $t+\Delta t$, this amount is removed by the surface reaction (1). The removed amount follows from the reaction rate $\eta_{\Gamma(t)}$ associated to (1) as

$\int_{S} \int_{\Gamma(\tau)} \eta_{\Gamma(\tau)}(x, \tau) \mathrm{d} \sigma \mathrm{d} \tau=\left|\Gamma\left(t^{\prime \prime}\right)\right| \Delta t \eta_{\Gamma\left(t^{\prime \prime}\right)}\left(x, t^{\prime \prime}\right)$

for some $t^{\prime \prime} \in(t, t+\Delta t]$. Therefore, we have

$\frac{\Delta s}{\Delta t}=\frac{\left|\Gamma\left(t^{\prime \prime}\right)\right|}{\left|\Gamma\left(t^{\prime}\right)\right|} \frac{1}{\phi \phi_{w} H\left(x, t^{\prime}\right)} \eta_{\Gamma\left(t^{\prime \prime}\right)}\left(x, t^{\prime \prime}\right)$.

Equation 3 essentially describes a situation in which $\mathrm{Ca}(\mathrm{OH})_{2}$ is completely transformed into $\mathrm{CaCO}_{3}$ (with rate $\left.\eta_{\Gamma(t)}\right)$. In other words, Eq. 3 may hold only in the case when molecules of $\mathrm{Ca}(\mathrm{OH})_{2}$ do not have time to be leached away by transport mechanisms. Letting $\Delta t \rightarrow 0$ on both sides of (3), we obtain for the velocity $s^{\prime}$ of $\Gamma(t)$ the expression 
$s^{\prime}(x, t)=\frac{1}{\phi \phi_{w} H(x, t)} \eta_{\Gamma(t)}(x, t), \quad x \in \Gamma(t), \quad t>0$.

Observe that the precise definition of $\eta_{\Gamma(t)}$ is not needed to derive (4). We expect that the denominator of (4) is strictly positive for all times, i.e.,

$\phi \phi_{w} H(s(t), t)>0 \quad$ for all $t \geq 0$.

Usual modifications of the argument in the case of more space dimensions $(n=2,3)$ and symmetric geometric configurations yield (4), too. Note that (4) gives (for the one-dimensional case) the penetration depth

$s(t)=s_{0}+\int_{0}^{t} \frac{1}{\phi \phi_{w} H(s(\tau), \tau)} \eta_{\Gamma(\tau)}(s(\tau), \tau) \mathrm{d} \tau$

for each $t \in S$.

Let us now remove the hypothesis that (1) is a surface-concentrated reaction and present a faute de mieux ansatz for the velocity of a reaction strip $\Omega_{\epsilon}(t)$ (having a sufficiently small width $\epsilon$ ). The range of validity of such a velocity law will depend not only on the size of $\epsilon$ but also on the correctness of the velocity for the sharp interface [where (1) is assumed to localize as $\epsilon$ goes to zero]. If we think now of moving a thin reaction strip $\Omega_{\epsilon}(t)$, then our ansatz for $s^{\prime}(t)$ is

$s^{\prime}(t)=c_{s}(\epsilon) \frac{\int_{\Omega_{\epsilon}(t)} \eta_{\Omega_{\epsilon}(t)}(x, t) \mathrm{d} x}{\int_{\Omega_{\epsilon}(t)} \phi \phi_{w} H(x, t) \mathrm{d} x}$,

where $\eta_{\Omega_{\epsilon}(t)}(x, t)$ denotes the carbonation reaction rate and now (1) is considered to take place within the strip $\Omega_{\epsilon}(t)$. The factor $c_{s}(\epsilon)$ is typically proportional to $\epsilon$ [9]. Equation 7 is a sort of dilation of (4). More modeling work is needed to obtain a better variant of (7). Note that the expression of the velocity $s^{\prime}$ (defined by the non-local law (7)) possesses a certain regularizing effect (compared to the sharp-interface case). The structure of (7) typically allows the quantity $\epsilon \eta_{\Omega_{\epsilon}(t)}$ to converge to $\eta_{\Gamma(t)}$ as $\epsilon$ tends to zero.

Having velocity descriptions like (4) or (7) available, we can obtain estimates on the final time of the process in a straightforward fashion using some ode-type arguments [9]. Let us discuss the case when (4) holds. The second case can be dealt with similarly. Let $T_{\text {fin }}$ be the time that (1) needs to fully carbonate the region $\left[s_{0}, L_{0}\right]$ (see Fig. 1). Solving (6) for $t$, we obtain for $t=T_{\text {fin }}$ the inequalities

$\frac{L_{0}-s_{0}}{\eta_{\max }}<T_{\text {fin }}<\frac{L_{0}-s_{0}}{\eta_{\min }}$.

In (8), $\eta_{\min }$ and $\eta_{\max }$ represent known quantitative lower and, respectively, upper bounds on the speed $s^{\prime}$ such that $\eta_{\min }<s^{\prime}(t)<\eta_{\max }$

for all $t>0$. The upper bound on $T_{\text {fin }}$ (see the r.h.s. of (8)) is an estimate of the time to initiate corrosion defined in [18], that is the time that the reaction interface, positioned at $x=s(t)$, needs to reach the point $x=L$, where the steel reinforcement is imbedded in the concrete wall.

In order to visualize how fast a reaction interface or strip can indeed move, one needs a PDE model to describe this. In Sect.4.2, we derive from first principles what such a model looks like for the case of a moving sharp carbonation interface. Clearly, if we wish to displace a reaction strip, the law (4) has to be replaced by (7).

\subsection{Derivation of a two-phase sharp-interface model}

The velocity law (4) together with

$s(0)=s_{0}>0$

describes the motion of the reaction interface $\Gamma(t)$ in the $1 \mathrm{D}$ case. To be more precise, we plan to follow the motion of the point $x=s(t)$ within the finite interval $\left[s_{0}, L\right]$. In order to obtain a complete model, balance equations for the 
active species as well as corresponding initial, boundary and transmission conditions have to be added. The resulting model will be nonlinear, non-local, strongly coupled, and will have PDEs defined on time-varying domains whose shape is a priori unknown. We denote by $C(x, t), D(x, t), B(x, t), H(x, t)$ and $W(x, t)$ the (averaged) mass concentrations of $\mathrm{CO}_{2}(\mathrm{aq}), \mathrm{CO}_{2}(\mathrm{~g}), \mathrm{CaCO}_{3}(\mathrm{aq}), \mathrm{Ca}(\mathrm{OH})_{2}(\mathrm{aq})$ and humidity. Recalling the basic geometry described in Fig. 1, let the carbonation region be $\Omega_{1}(t):=(0, s(t))$, while the uncarbonated region is $\Omega_{2}(t):=(s(t), L)$. Clearly, we then have $\Omega:=\Omega_{1}(t) \cup\{x=s(t)\} \cup \Omega_{2}(t)$ for any time $t \geq 0$. The corresponding mass-balance equations have the structure

$$
\begin{aligned}
& C_{t}-D_{c} C_{x x}=P(Q D-C) \text { in } \Omega_{1}(t), \\
& D_{t}-D_{d} D_{x x}=-P(Q D-C) \text { in } \Omega_{1}(t), \\
& H_{t}-D_{h} H_{x x}=S_{\mathrm{diss}}\left(H_{\mathrm{eq}}-H\right) \text { in } \Omega_{2}(t), \\
& B_{t}-D_{b} B_{x x}=S_{\mathrm{prec}}\left(B-B_{\mathrm{eq}}\right) \text { in } \Omega_{1}(t), \\
& W_{t}-D_{w} W_{x x}=0 \text { in } \Omega,
\end{aligned}
$$

where $D_{c}, D_{d}, D_{h}$ and $D_{w}$ are effective transport parameters and $Q, H_{\mathrm{eq}}, B_{\mathrm{eq}}, P, S_{\mathrm{prec}}$ and $S_{\mathrm{diss}}$ are, respectively, Henry's constant, equilibrium concentrations of aqueous $\mathrm{Ca}(\mathrm{OH})_{2}$ and $\mathrm{CaCO}_{3}$ and mass-transfer coefficients. The Henry constant for $\mathrm{CO}_{2}$ is known, while the equilibrium concentrations $H_{\text {eq }}$ and $B_{\text {eq }}$ are estimated by calculating how much $\mathrm{Ca}(\mathrm{OH})_{2}$ (resp. $\mathrm{CaCO}_{3}$ ) can be absorbed in the pore water. These two parameters can be determined exactly by extending the current model into a PDE system that accounts for ionic transport mechanisms, equilibrium redox/ionic reactions and electrical charge conservation. The balance equations (10)-(11) allow the transfer of $\mathrm{CO}_{2}$ from the air-filled parts of the pores into the pore water, while (12) and (13) incorporate the dissolution of $\mathrm{Ca}(\mathrm{OH})_{2}$ in the pore water and the precipitation of $\mathrm{CaCO}_{3}$ to the solid matrix.

The chemistry of the cement [12] is fully described by the initial conditions

$E(x, 0)=E_{0}$,

where the species $E$ denotes $B, C, D, H$ and $W$, respectively. By the same simplifying notation, the isolation conditions at $x=L$ can be expressed as

$E_{x}(L, t)=0$,

where now $E=H, W$. At the outer boundary $x=0$, we have the following conditions.

$C(0, t)=\lambda_{c}, D(0, t)=\lambda_{d}, B_{x}(0, t)=W_{x}(0, t)=0$.

In (17), we specify $\lambda_{c}$ and $\lambda_{d}$ in order to be in agreement with either accelerated or natural exposure tests, where the sample is sheltered from rain. If the sample is exposed to rain, essentially different types of boundary conditions need to be imposed; see [31] for related comments. Furthermore, the concentrations $B, C, H$, and $W$ (together with their gradients) need to satisfy Rankine-Hugoniot jump relations ${ }^{3}$ at $x=s(t)$. These relations are typically obtained via a shrinking pillbox argument as mentioned, for instance, in [32]. We have

$$
\begin{aligned}
& -D_{c} C_{x}(s(t), t)=s^{\prime}(t) C(s(t), t)+\eta_{\Gamma(t)}(s(t), t), \\
& -D_{d} D_{x}(s(t), t)=s^{\prime}(t) D(s(t), t), \\
& -D_{b} B_{x}(s(t), t)=0, \\
& -D_{h} H_{x}(s(t), t)=s^{\prime}(t) H(s(t), t)-\eta_{\Gamma(t)}(s(t), t), \\
& {\left[-D_{w} W_{x}\right]_{x=s(t)}=-\eta_{\Gamma(t)}(s(t), t),[W]_{x=s(t)}=0,}
\end{aligned}
$$

where $[E]_{x=s(t)}$ denotes the jump in $E(x, t)$ at the position $x=s(t)$. In this scenario, the reaction rate is assumed to have the nonlinear form

$\eta_{\Gamma(t)}(s(t), t)=k C(x, t)^{p} H(x, t)^{q}$ for $x=s(t)$,

\footnotetext{
$\overline{3}$ The role of the Rankine-Hugoniot conditions is to ensure the conservation of mass across $\Gamma(t)$.
} 
for all $t \geq 0$. In (23), $k$ represents the reaction constant while $p$ and $q$ are partial reaction exponents. Here, $k, p$ and $q$ are strictly positive, while for mathematical simplicity we take $p \geq 1$ and $q \geq 1$.

The system (9-23) forms a kinetic moving-boundary model. Additionally, if we assume that the species $B$ precipitates to the porous matrix and is not further diffusing, then (13) becomes an ODE. Hence boundary and interface conditions for $B$ are no longer required.

\section{Nonlinear effects by reaction}

In this section, we illustrate that the MBP derived in Sect. 4.2 captures specific effects induced by the carbonation reaction. Here we only mention some of them. Detailed information concerning simulation issues as well as fairly complete descriptions of the involved cement chemistry are reported, for instance in $[9,16,17,23,33]$.

\subsection{Simulation examples}

For the numerical simulations we rely on two different experimental observations:

(a) accelerated carbonation tests, i.e., short-term tests for which most of the data is fairly well controlled;

(b) natural carbonation tests, i.e., long-term tests for which the exact magnitude of some of the model parameters is unknown.

Observe that the output of the moving-boundary models (models of types 1-3) consists of the penetration depth vs. time curve and of the concentration profiles, while the output of isoline models (models of type 4) only consists of concentration profiles. The next plots contain effects discovered by means of models of types $1-4$. All of them suggest the presence of a reaction zone of a particular strength (with a high Thiele modulus $[9,14,17]$ ) somewhere in the interior of $\Omega$. These internal zones (either strips or sharp interfaces) are noticed in the carbonation case mainly due to the fact that the characteristic time of reaction (1) is much smaller than that of the transport of $\mathrm{CO}_{2}(\mathrm{~g})$.

In Fig. 2, we show an example where two internal reaction strips (instead of one) occur. The strips correspond to different carbonation-type reactions. More precisely, in this figure it is about the simultaneous carbonation of $\mathrm{Ca}(\mathrm{OH})_{2}$ via (1) and the carbonation of CSH-phases (see [12] for the exact meaning of the notation as well as the chemical properties of the compound and [13] for a more mathematical description of CSH-phases formation through hydration reactions)

$\mathrm{C}_{\mathrm{x}} \mathrm{S}_{\mathrm{y}} \mathrm{H}_{\mathrm{z}}+\mathrm{xH}_{2} \mathrm{CO}_{3} \rightarrow \mathrm{xCaCO}_{3}+\mathrm{ySiO}_{2} \cdot \mathrm{tH}_{2} \mathrm{O}+(\mathrm{x}-\mathrm{t}+\mathrm{z}) \mathrm{H}_{2} \mathrm{O}$.

Simulation details as well as the carbonation experiment employed are explained in [17]. Note that the second strip has a lower amplitude and a larger width than the first. Moreover, the two strips are almost non-overlapping. This fact suggests the following scenario: As soon as (1) is finished, (24) goes to completion. Reaction (24) indicates that a production by secondary carbonation can take place behind the strip $\Omega_{\epsilon}(t)$, namely in $\Omega_{1}(t)$. Another secondary carbonation effect (this time induced by a secondary dissolution of $\mathrm{Ca}(\mathrm{OH})_{2}$ ) is pointed out in Fig. 3. Here, two internal zones of depletion of aqueous $\mathrm{Ca}(\mathrm{OH})_{2}$ can be observed. The first zone corresponds (again) to $\Omega_{1}(t)$, while the second one is $\Omega_{\epsilon}(t)$. Carbonation within $\Omega_{1}(t)$ slows down the penetration speed of the front; it acts like a retarder with respect to the overall carbonation penetration. Typical concentration profiles and penetration depths (obtained with a model of type 3) are shown in Figs. 4 and 5.

In Fig. 6, the water-barrier effect is pointed out; see [23]. In fact, we note that the production of water by (1) may locally fill air parts of the pores, thus hindering the transport of $\mathrm{CO}_{2}$ in the air phase of the pores. This effect is visible when simulating accelerated tests in which the $\mathrm{CO}_{2}$ concentration in the carbonation chamber is too high. Hence, high production rates of water and $\mathrm{CaCO}_{3}$ are expected and a localized water front (acting as a barrier) or a partial clogging of the pores with $\mathrm{CaCO}_{3}$ can indeed happen. Preliminary remarks on limitations in the use of accelerated concrete-carbonation tests for service-life predictions are presented in [35]. The water-barrier effect raises some 


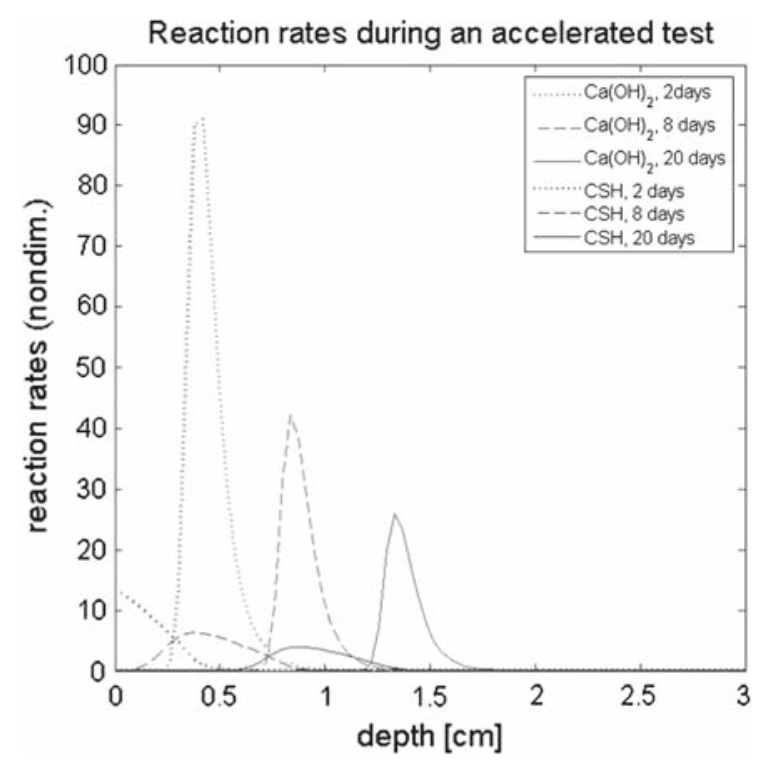

Fig. 2 Internal strip formation in a concrete carbonation scenario with two carbonatable components. Both $\mathrm{Ca}(\mathrm{OH})_{2}$ and $\mathrm{CSH}$ react with $\mathrm{CO}_{2}$ to produce carbonates [17] (cf. model of type 4)

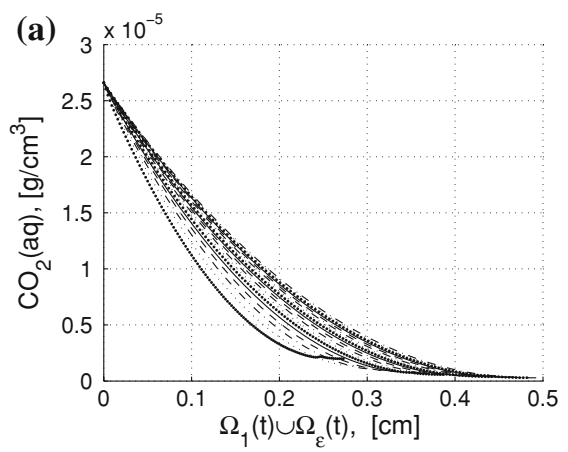

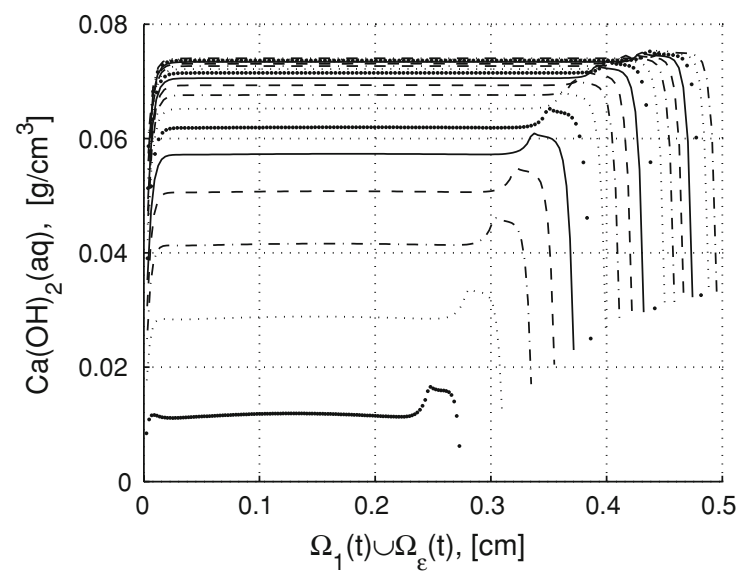

Fig. 3 Effect of secondary carbonation (cf. model of type 3): Two internal zones of depletion of $\mathrm{Ca}(\mathrm{OH})_{2}(\mathrm{aq})$ (the flat curve corresponds to $\Omega_{1}(t)$ while the bump is $\left.\Omega_{\epsilon}(t)\right)$ are formed and progress into the material. Each curve refers to time $t=i$ years, $i \in\{1, \ldots, 18\}$

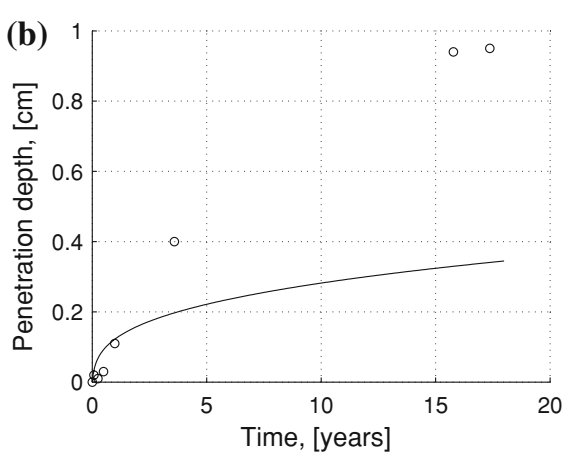

Fig. 4 a $\mathrm{CO}_{2}(\mathrm{aq})$ and $\mathrm{CO}_{2}(\mathrm{~g})$ concentration profiles $v s$. space. Each curve refers to time $t=i$ years, $i \in\{1, \ldots, 18\}$. b Interface position $v s$. the experimental points "o" after $T_{\text {fin }}=18$ years of exposure

doubts about the relevance of many accelerated laboratory experiments at high $\mathrm{CO}_{2}$ concentration. Since a huge amount of experimental data already exists, the mathematical challenge is not to deny the usefulness of accelerated tests, but rather to try to extract some qualitative information out of them. A first step could be an attempt to better understand the intimate interplay between the production of water by (1), water transport in porous media, water as host of carbonation reaction, and the change in porosity and water fraction induced by (1), especially concerning the long-time behavior of active concentrations.

\subsection{Limitations of the kinetic moving-boundary approach}

Effects on penetration depths induced by special geometries of the samples can be captured with models of type 4 by employing an adaptive finite-element strategy; see [23]. In this class of models, the production terms by 

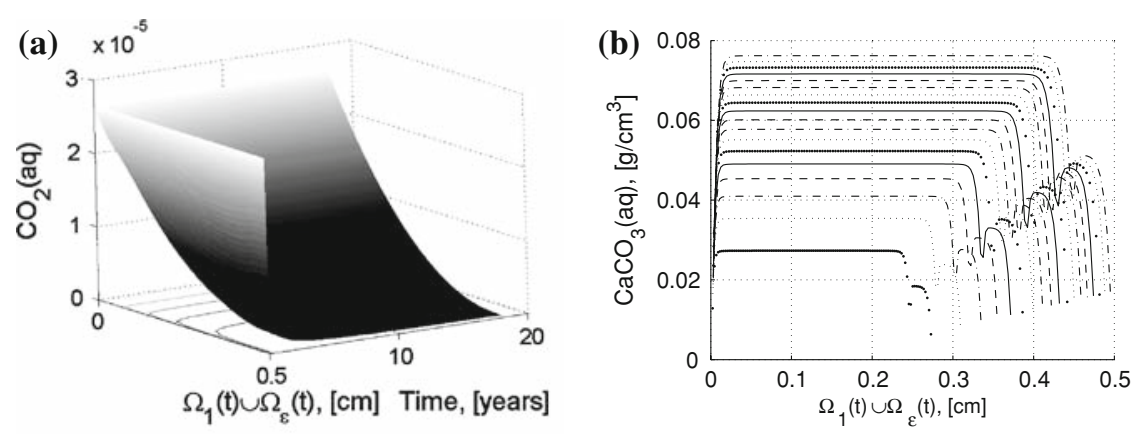

(c)
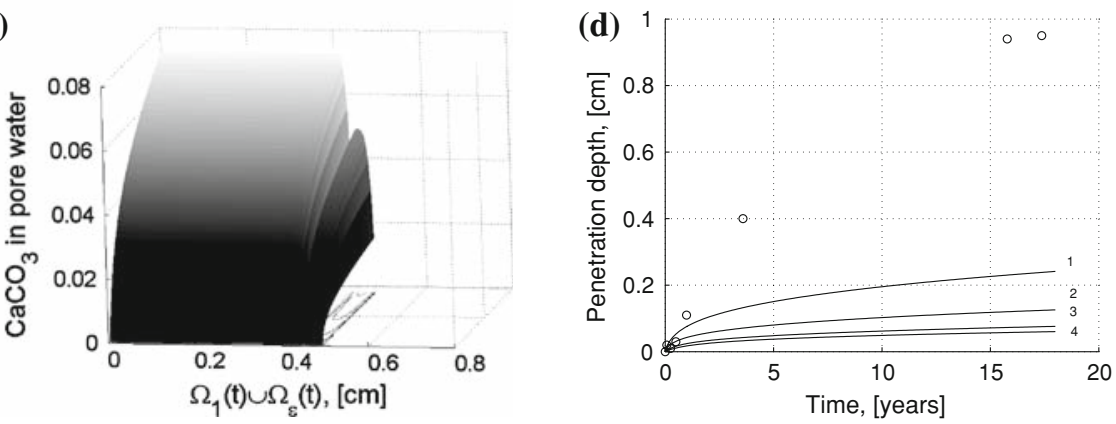

Fig. 5 a Concentration profiles of $\mathrm{CO}_{2}(\mathrm{aq}) v s$. time and space position. $\mathbf{b} \mathrm{CaCO}_{3}(\mathrm{aq})$ concentration profiles $v s$. space. Each curve refers to time $t=i$ years, $i \in\{1, \ldots, 18\}$. c Two internal zones of production of $\mathrm{CaCO}_{3}$ develop into the material (see the counter-piece in Fig. 3). d Increasing of the strength of the reaction rate in $\Omega_{1}(t)$, when the reaction rate associated to $\Omega_{\epsilon}(t)$ is kept fixed, [9]
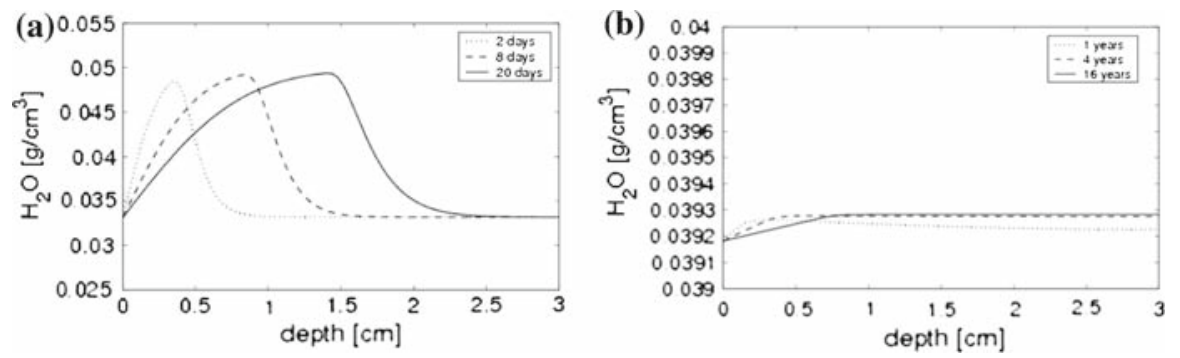

Fig. 6 Prediction of moisture profiles under accelerated and natural conditions, see [35]. a accelerated test, $\mathrm{CO}_{2}=50 \%$, $\mathrm{RH}=60 \%$; b natural exposure test, $\mathrm{CO}_{2}=0.03 \%, \mathrm{RH}$ (relative humidity) $=78 \%$

reaction (1) enter the r.h.s of the mass-balance equations for $C, H, B$, and $W$. It is worth mentioning that in the term $k C^{p} H^{q}$ the reaction constant $k$ is of the order of $\mathcal{O}\left(\frac{1}{\epsilon}\right)$. Consequently, as $\epsilon$ tends to zero, this term enforces the spatial segregation of $C, D$, and $B$ from $H$ [36]. Two simulation examples are shown in Figs. 7 and 8 for the case of the experimental data from [34]. Figure 7 shows concentration fields of $\mathrm{CO}_{2}(\mathrm{~g}), \mathrm{CO}_{2}(\mathrm{aq})$ and $\mathrm{Ca}(\mathrm{OH})_{2}$ at two different times during simulation. Note that after a few days of carbonation, a clear separation between the carbonated region and the uncarbonated one appears. After an initial transient time typically of order of $\mathcal{O}(\epsilon)$ (cf. Remark 1 in [37]), a thin reaction strip is formed and is ready to move. It appears that near the convex outer corners, the carbonation strip progresses faster than at straight edges, and it moves even slower near the concave inner corner.

The last two figures have been obtained with the adaptive finite-element toolbox ALBERTA [24]. It is worth mentioning that models of types 1-3 have not yet been numerically explored in the (self-)adaptive framework. 

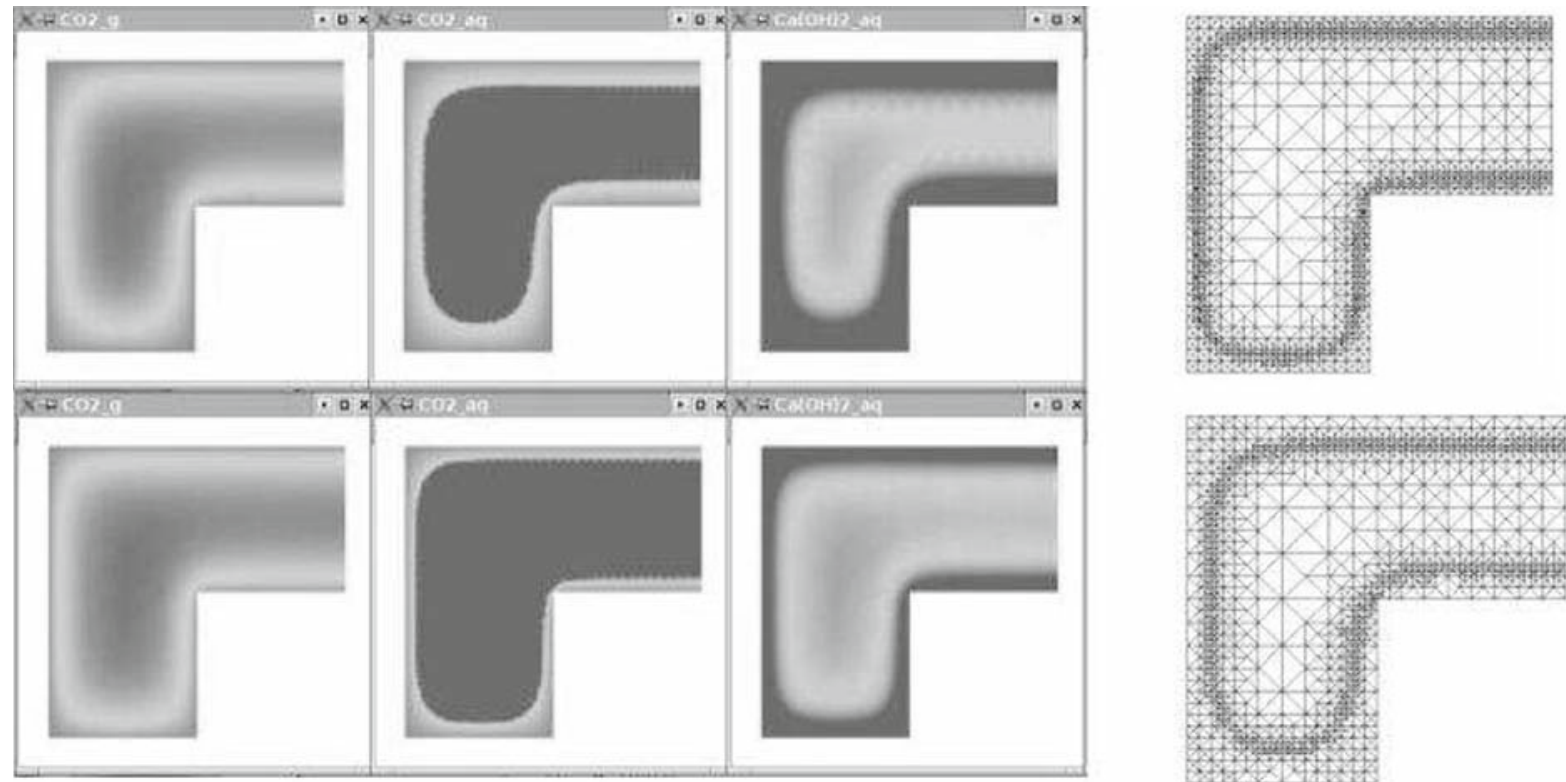

Fig. 7 Carbonation attack around a corner: self-adaptive numerical approach by means of a model of type 4; see [23]

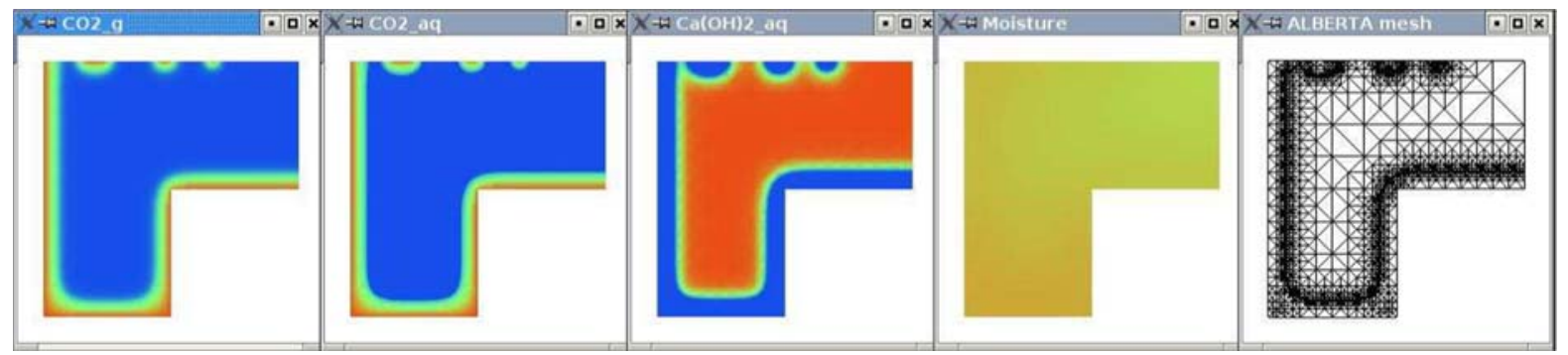

Fig. 8 Carbonation attack around a corner and wall holes after 250 days of natural exposure to $\mathrm{CO}_{2}$ : self-adaptive numerical approach by means of a model of type 4 , see [23]

\section{Discussion}

The list of new research directions arising from the study of the moving-boundary models of kinetic type (applied to carbonation or to similar physico-chemical processes) is open. We only mention a few aspects, which we believe to be relevant for the further understanding of the matter:

1. By dimensional inspection, one can observe that the width $\epsilon$ of the reaction strip $\Omega_{\epsilon}(t)$ is inversely proportional to the ratio of the characteristic reaction time and the characteristic diffusion time [9]. Specifically, the zone of reaction in such a case is narrowly confined to the interface separating the uncarbonated from the carbonated phases (like a strip). By this proportionality, we expect the strip to shrink to the interface $\Gamma(t)$ when conveniently altering that ratio, i.e., $\Omega_{\epsilon}(t) \rightarrow \Gamma(t)$ as $\epsilon \rightarrow 0$. Similar dimensional arguments suggest that the speed of the reaction strip is directly proportional to $1 / \epsilon$. Motivated by the results reported in [38-40], we expect that algebraic connections between $\epsilon$ and the partial reaction orders $p$ and $q$ (see (23)) can be obtained for models of types 1-3 at least for large times. A singular perturbation study may recover the sharp interface setting via shrinking the reaction strip as $\epsilon$ goes to zero.

2. We reported results of numerical simulations for various model situations. Most of the examples are given for one-dimensional domains. The extension of the models (of types 1-3) can be done with minimal changes to the $3 \mathrm{D}$ case for a moving planar sharp interface or for symmetric geometries like spheres and cylinders. The 
models of type 4 do not depend on the choice of the space dimension. However, the central question: "How can kinetic moving-boundary models be formulated in the case of several space dimensions?", still remains open. The main difficulty in the multidimensional case consists in finding the right description of the velocity of the sharp interface under the action of surface-concentrated reactions. In such scenarios, corners and straight edges are expected to play a significant role. At this point, we are wondering how we could incorporate the right geometry effects in the kinetic condition. Singular perturbation techniques seem to suggest that a small curvature term must be introduced. How small should this term be? Are there any other geometrical factors that need to be accounted for? A geometric phase-field approach (see for instance [41] for hints on the methodology) or an appropriate singular-limit analysis may help in guessing the natural description of the velocity in the 2D case in the presence of a corner.

3. The moving-boundary model can be extended to account for reaction-induced changes in porosities and water and air fractions. Note that, in such situations, the resulting PDE system (with or without moving interfaces) degenerates, and consequently, there are many challenges for understanding its mathematical analysis.

Acknowledgements We acknowledge the financial support of the German Science Foundation (DFG) under the grant SPP 1122 Prediction of the course of physicochemical damage processes involving mineral materials. The authors thank the two reviewers for their comments and suggestions that essentially contributed to the final version of the paper.

Open Access This article is distributed under the terms of the Creative Commons Attribution Noncommercial License which permits any noncommercial use, distribution, and reproduction in any medium, provided the original author(s) and source are credited.

\section{References}

1. Ortoleva P (1994) Geochemical self-organization. OUP, Oxford

2. Ball JM, Knderlehrer D, Podio-Guidugli P, Slemrod M (eds) (1999) Evolving phase interfaces in solids. Springer, Berlin

3. Tilley BS, Kriegsmann GA (2001) Microwave-enhanced chemical vapor infiltration: a sharp interface model. J Eng Math 534:971989

4. Dewynne JN, Fowler AC, Hagan PS (1993) Multiple reaction fronts in the oxidation-reduction of iron-rich uranium ores. SIAM J Appl Math 534:971-989

5. Visintin A (1986) A new model for supercooling and superheating effects IMA. J Appl Math 36:141-157

6. Evans JD, King JR (1996) On the derivation of heterogeneous reaction kinetics from a homogeneous reaction model. SIAM J Appl Math 60(6):1977-1996

7. Clarelli F, Fasano A, Natalini R (2008) Mathematics and monuments conservation: free boundary models of marble sulphation. SIAM J Appl Math 69(1):149-168

8. Muntean A, Böhm M (2006) Dynamics of a moving reaction interface in a concrete wall. In: Rodrigues JF et al (eds) Free and moving boundary problems. Theory and applications. Proceedings of the conference FBP 2005, Int Ser Numer Math, vol 154. Birkhäuser, Basel, pp 317-326

9. Muntean A (2006) A moving-boundary problem: modeling, analysis and simulation of concrete carbonation. Cuvillier Verlag, Göttingen

10. Muntean A (2007) Concentration blow up in a two-phase non-equilibrium model with source term. Meccanica 42:409-411

11. Muntean A (2009) Well-posedness of a moving-boundary problem with two moving reaction strips. Nonlinear Anal Real World Appl 10(4):2541-2557

12. Taylor HFW (1997) Cement chemistry. Thomas Telford, London

13. Salhan A, Billingham J, King AC (2003) The effect of a retarder on the early stages of the hydration of calcium silicate. J Eng Math 45:367-377

14. Papadakis VG, Vayenas CG, Fardis MN (1989) A reaction engineering approach to the problem of concrete carbonation. AIChE J 35:1639-1650

15. Lagerblad B (2005) Carbon dioxide uptake during concrete life cycle-State of the art. NI-project 03018, Swedish Cement and Concrete Research Institute, Stockholm

16. Peter M, Muntean A, Meier S, Böhm M (2008) Competition of several carbonaton reactions: a parametric study. Cement Concr Res 38:1385-1393

17. Meier SA, Peter MA, Muntean A, Böhm M (2007) Dynamics of the internal reaction layer arising during carbonation of concrete. Chem Eng Sci 62:1125-1137

18. Saetta AV, Schrefler BA, Vitaliani RV (1995) 2D model for carbonation, moisture/heat flow in porous materials. Cement Concr Res 32:939-941 
19. Matkowsky BJ, Sivashinsky GI (1979) An asymptotic deriavtion of two models in flame theory associated with the contant density approximation. SIAM J Appl Math 37(3):686-699

20. Matkowsky BJ, Sivashinsky GI (1978) Propagation of a pulsating reaction front in solid fuel combustion. SIAM J Appl Math 35(3):465-478

21. Chaussadent T (1999) États des lieux et réflexions sur la carbonatation du beton armé. LCPC, Paris

22. Böhm M, Kropp J, Muntean A (2003) A two-reaction-zones moving-interface model for predicting $\mathrm{Ca}(\mathrm{OH})_{2}$ carbonation in concrete. Berichte aus der Technomathematik, University of Bremen, 03-04

23. Schmidt A, Muntean A, Böhm M (2007) Moving carbonation fronts around corners: a self-adaptive finite element approach. In: 5th International Essen Workshop, Transcon, pp 467-476

24. Schmidt A, Siebert KG (2005) Design of adaptive finite element software: the finite element toolbox ALBERTA. LNCSE Series 42 Springer, Verlag, Berlin

25. Froment G, Bischoff KB (1996) Chemical reactor analysis and design. Wiley, New York

26. Tuutti K (1982) Corrosion of steel in concrete. Swedish Cement and Concrete Research Institute (CBI), Stockholm

27. Meirmanov A (1992) The Stefan problem. Walter de Gruyter, Berlin

28. Stefan J (1890) Über die Theorie der Eisbildung. Monatshefte der Mathematik und Physik 1(1):1-6

29. Alexiades V, Solomon A (1993) Mathematical modeling of melting and freezing processes. Hemisphere, Washington

30. Chalmers B (1977) Principles of solidification. Krieger, New York

31. Borsi I, Farina A, Primicerio M (2006) A rain infiltration model with unilateral boundary condition: qualitative analysis and numerical simulations. Math Methods Appl Sci 29:2047-2077

32. Gurtin M (1992) Thermomechanics of evolving phase boundaries in the plane. Clarendon, Oxford

33. Bunte D (1994) Zum Karbonatisierungsbedingten Verlust der Dauerhaftigkeit von Aussenbauteuilen aus Stahlbeton. Dissertation, TU Braunschweig

34. Steffens A, Dinkler D, Ahrens H (2002) Modeling carbonation for corrosion risk prediction of concrete structures. Cement Concr Res 32:935-941

35. Muntean A, Meier S, Peter M, Böhm M, Kropp J (2006) A note on the limitations of the use of accelerated concrete-carbonation tests for service life predictions. Berichte aus der Technomathematik, University of Bremen, 04-06

36. Crooks ECM, Dancer EN, Hilhorst D, Mimura M, Ninomiya H (2004) Spatial segregation limit of a competition-diffusion system with Dirichlet boundary conditions. Nonlinear Anal Real World Appl 5:645-665

37. Seidman TI (2009) Interface conditions for a singular reaction-diffusion system. Discret Contin Dyn Syst (to appear)

38. Bazant MZ, Stone HA (2000) Asymptotics of reaction-diffusion fronts with one static and one diffusing reactant. Physica D 147:95-121

39. Muntean A (2008) On the interplay between fast reaction and slow diffusion in the concrete carbonation process: a matched-asymptotics approach. Meccanica 44(1):35-46

40. Fila M, Souplet P (2001) Existence of global solutions with slow decay and unbounded free boundary for a superlinear Stefan problem. Interfaces Free Bound 3:1089-1112

41. Fife PC (1988) Dynamics of internal layers and diffusive interfaces. SIAM, Philadelphia 